Research Article

\title{
Generalizations of Fixed-Point Theorems of Altman and Rothe Types
}

\author{
Li Sun, ${ }^{1,2}$ Jingxian Sun, ${ }^{2}$ and Guangwa Wang ${ }^{2}$ \\ ${ }^{1}$ School of Mechanics and Civil Engineering, China University of Mining \& Technology, Xuzhou, Jiangsu 221008, China \\ ${ }^{2}$ School of Mathematics and Statistics, Jiangsu Normal University, Xuzhou, Jiangsu 221116, China \\ Correspondence should be addressed to Guangwa Wang; wanggw7653@163.com
}

Received 19 June 2013; Revised 9 September 2013; Accepted 27 September 2013

Academic Editor: Lishan Liu

Copyright (c) $2013 \mathrm{Li}$ Sun et al. This is an open access article distributed under the Creative Commons Attribution License, which permits unrestricted use, distribution, and reproduction in any medium, provided the original work is properly cited.

It is intended to present some extensions of the famous Altman and Rothe types fixed-point theorems. The inequality conditions are relaxed to the $\alpha$-positive-homogeneous operator. Some new fixed-point theorems are obtained with the help of the theory of topological degree.

\section{Introduction}

The topological degree theory and fixed-point theorems play an important role in the study of fixed points for various classes of nonlinear operators in Banach spaces. Many important results in mathematics are obtained by the use of these theories (see [1-12]). For example, Altman theorem is an important result from the theoretical as well as the applied point of view and is one of the core issues of mathematics. This fixed-point theorem has plenty of extensions and generalizations in the framework of nonlinear functional analysis and finds a wide application. In recent years, some researchers have focused on the inequality condition in Altman theorem and gained a lot of different generalized forms (see [26]). In this paper, we continue to study Altman theorem and relax the condition of square function to $\alpha$-positivehomogeneous operator. It is well known that $\alpha$-positivehomogeneous operator is a class of important nonlinear operators. Linear operator is an $\alpha$-positive-homogeneous operator when $\alpha=1$. Our main aim is to present and prove a collection of new fixed-point theorems for $\alpha$-positivehomogeneous operator. Many existing results in the references are extended. On the other hand, it is well known that semiclosed 1-set-contraction mapping is a significant operator and research object in nonlinear functional analysis (see $[3-5,7,8])$. Clearly, this class of 1-set-contractive operators includes strict set-contractive operators, condensing operators, semicontractive operators, completely continuous operators, and some others. The fixed-point theory of 1-setcontractive operators plays an important role in the study of the existence of the solution of some operator equations. Inspite of this, completely continuous operators still have a pivotal position in modern nonlinear functional analysis. They have a widely and profoundly applicative prospect. Therefore, in this paper, we mainly investigate the fixedpoint theorems about completely continuous operators and get some new results. Our conclusions are different from the ones in many recent works and extend some commonly known theorems. As far as we know, the cases in our paper have scarcely been seen in the available reference materials.

This paper contains three sections besides the introductory one. In Section 2, we give some basic concepts and some preparatory theorems. The main results will be presented and proved in Section 3. Then, we will list some existing results in the reference as our corollaries and this is the content of Section 4.

\section{Preliminaries}

In this section, we review some basic concepts and results which will be used later. For convenience, throughout all the paper, we let $E, F$ be two real Bananch spaces and let $\Omega$ be a bounded open subset of $E$ and $\theta \in \Omega$. 
Definition 1 ( $\alpha$-positive-homogeneous operator). Given $\alpha \epsilon$ $\mathbb{R}$, the mapping $B: E \rightarrow F$ is called an $\alpha$-positivehomogeneous operator if for any $x \in E, t \geqslant 0$, the equation

$$
B(t x)=t^{\alpha} B x
$$

always holds.

Definition 2 (cone). If $P$ is a subset of Banach space $F$ and satisfies the following three conditions:

(i) $P$ is a closed and convex set;

(ii) $x \in P, \alpha \geqslant \theta \Rightarrow \alpha x \in P$;

(iii) $x \in P, x \neq \theta \Rightarrow-x \notin P$,

then $P$ is called a cone in $F$. Furthermore, if $x, y \in F$, then $y-x \in P$ defines a semiorder, denoted by $x \leqslant y$.

Lemma 3 (see [9], Leray-Schauder). Suppose that $A: \bar{\Omega} \rightarrow$ $E$ is a completely continuous operator. If

$$
A x \neq \mu x, \quad \forall x \in \partial \Omega, \mu \geqslant 1,
$$

then $\operatorname{deg}(I-A, \Omega, \theta)=1$, and hence $A$ has at least one fixed point in $\bar{\Omega}$.

Lemma 4 (see $[3,5]$, Leray-Schauder). Suppose that $A: \bar{\Omega} \rightarrow$ $E$ is a semiclosed 1-set-contractive operator; that is, $A$ is 1-set contraction and $I-A$ is closed. If

$$
A x \neq \mu x, \quad \forall x \in \partial \Omega, \mu \geqslant 1,
$$

then $\operatorname{deg}(I-A, \Omega, \theta)=1$, and hence $A$ has at least one fixed point in $\bar{\Omega}$.

In Lemmas 3 and 4 , the same condition that

$$
A x \neq \mu x, \quad \forall x \in \partial \Omega, \mu \geqslant 1
$$

ia called (L-S) condition.

Theorem 5 (see [9]). Suppose that $A: \bar{\Omega} \rightarrow E$ is a completely continuous operator. If one of the following condition is satisfied:

(i) (Altman) $\|A x-x\|^{2} \geqslant\|A x\|^{2}-\|x\|^{2}$, for all $x \in \partial \Omega$,

(ii) (Rothe) $\|A x\| \leqslant\|x\|$, for all $x \in \partial \Omega$,

(iii) (Petryshyn) $\|A x\| \leqslant\|A x-x\|$, for all $x \in \partial \Omega$,

then $\operatorname{deg}(I-A, \Omega, \theta)=1$, and hence $A$ has at least one fixed point in $\bar{\Omega}$.

Theorem 6 (see [5]). Suppose that $A: \bar{\Omega} \rightarrow E$ is a semi-closed 1-set-contractive operator. If one of the following conditions is satisfied:

(i) (Altman) $\|A x-x\|^{2} \geqslant\|A x\|^{2}-\|x\|^{2}$, for all $x \in \partial \Omega$,

(ii) (Rothe) $\|A x\| \leqslant\|x\|$, for all $x \in \partial \Omega$,

(iii) (Petryshyn) $\|A x\| \leqslant\|A x-x\|$, for all $x \in \partial \Omega$,

then $\operatorname{deg}(I-A, \Omega, \theta)=1$, and hence $A$ has at least one fixed point in $\bar{\Omega}$.

\section{Main Results}

Now, we present and prove our main results.

Theorem 7. Suppose that $A: \bar{\Omega} \rightarrow E$ is a completely continuous operator. Let $P$ be a cone of $F$ and let the operator $B: E \rightarrow$ $P$ be $\alpha$-positive-homogeneous such that $B x>\theta$ for $x \neq \theta$. If $\alpha>1$ and $B$ satisfies the following condition:

$$
B(A x-x) \geqslant B A x-B x, \quad \forall x \in \partial \Omega,
$$

then $A$ has at least one fixed point in $\bar{\Omega}$.

Proof. If the operator $A$ has a fixed point on $\partial \Omega$, then $A$ has at least one fixed point in $\bar{\Omega}$. Now, suppose that $A$ has no fixed points on $\partial \Omega$. It suffices to prove that (5) implies the condition (L-S). Suppose the contrary, then there exist $x_{1} \in \partial \Omega$ and $\mu_{1} \geqslant 1$ such that $A x_{1}=\mu_{1} x_{1}$; it is easy to see that $\mu_{1}>1$.

Now, consider the function defined by

$$
f(t)=(t-1)^{\alpha}-t^{\alpha}+1, \quad \forall t \geqslant 1, \alpha>1 .
$$

Since $f^{\prime}(t)=\alpha(t-1)^{\alpha-1}-\alpha t^{\alpha-1}<0$ by formal differentiation, $f(t)$ is strictly decreasing in $[1,+\infty)$, and so $f(t)<f(1)=0$, for all $t>1$; that is, $(t-1)^{\alpha}<t^{\alpha}-1$, for all $t>1, \alpha>1$. Then, we can get from (5)

$$
\begin{aligned}
B\left(A x_{1}-x_{1}\right) & =B\left(\mu_{1} x_{1}-x_{1}\right) \\
& =\left(\mu_{1}-1\right)^{\alpha} B x_{1} \\
& \geqslant B A x_{1}-B x_{1} \\
& =B\left(\mu_{1} x_{1}\right)-B x_{1} \\
& =\mu_{1}^{\alpha} B x_{1}-B x_{1} \\
& =\left(\mu_{1}^{\alpha}-1\right) B x_{1} ;
\end{aligned}
$$

that is, $\left(\mu_{1}-1\right)^{\alpha} B x_{1} \geqslant\left(\mu_{1}^{\alpha}-1\right) B x_{1}$. Thus $\left[\left(\mu_{1}-1\right)^{\alpha}-\left(\mu_{1}^{\alpha}-\right.\right.$ 1)] $B x_{1} \doteq \alpha_{1} B x_{1} \in P$. Noticing $\alpha_{1}<0$ and $B x_{1} \neq \theta$, one can has that this contradicts the condition (iii) in Definition 2, and so the condition (L-S) is satisfied. Therefore, it follows from Lemma 3 that the conclusion of Theorem 7 holds.

Remark 8. Obviously, we only need to take $B=\|\cdot\|^{2}$ in Theorem 7 and we can get the famous fixed-point theorem of Altman in Theorem 5 (i). Consequently, Theorem 7 generalizes the classical Altman theorem.

Theorem 9. Suppose that $A: \bar{\Omega} \rightarrow E$ is a completely continuous operator. Let $P$ be a cone of $F$ and let the operator $B$ : $E \rightarrow P$ be $\alpha$-positive-homogeneous such that $B x>\theta$ for $x \neq \theta$. If $\alpha>1$ and $B$ satisfies the following condition:

$$
B A x \leqslant B x, \quad \forall x \in \partial \Omega,
$$

then $A$ has at least one fixed point in $\bar{\Omega}$.

Proof. We will also prove that (L-S) is satisfied under the condition (8). Suppose this is not true. Then there exist 
$x_{2} \in \partial \Omega$ and $\mu_{2} \geqslant 1$ such that $A x_{2}=\mu_{2} x_{2}$. It is easy to see that $\mu_{2}>1$. Then we can get from (8)

$$
B\left(A x_{2}\right)=B\left(\mu_{2} x_{2}\right)=\mu_{2}^{\alpha} B x_{2} \leqslant B x_{2} ;
$$

that is, $B x_{2} \geqslant \mu_{2}^{\alpha} B x_{2}$, thus $\left(1-\mu_{2}^{\alpha}\right) B x_{2} \doteq \alpha_{2} B x_{2} \in P$. This contradicts the condition (iii) in Definition 2 on account of $\alpha_{2}<0, B x_{2} \neq \theta$, and so the condition (L-S) is satisfied. Therefore, it follows from Lemma 3 that the conclusion of Theorem 9 holds.

Remark 10. Taking $B=\|\cdot\|^{2}$ in Theorem 9, we can obtain the Rothe theorem in Theorem 5 (ii) immediately.

Theorem 11. Suppose that $A: \bar{\Omega} \rightarrow E$ is a completely continuous operator. Let $P$ be a cone of $F$ and let $B: E \rightarrow P$ be an $\alpha$-positive-homogeneous operator such that $B x>\theta$ for $x \neq \theta$. If $\alpha>1$ and $B$ satisfies the following condition:

$$
B A x \leqslant B(A x-x), \quad \forall x \in \partial \Omega,
$$

then $A$ has at least one fixed point in $\bar{\Omega}$.

Proof. Similarly, we will also prove that (L-S) is satisfied. Suppose the contrary, then there exist $x_{3} \in \partial \Omega$ and $\mu_{3} \geqslant 1$ such that $A x_{3}=\mu_{3} x_{3}$. Obviously, $\mu_{3}>1$. Then we can get from (10)

$$
\begin{aligned}
B\left(A x_{3}\right) & =B\left(\mu_{3} x_{3}\right)=\mu_{3}^{\alpha} B x_{3} \\
& \leqslant B\left(A x_{3}-x_{3}\right)=B\left(\mu_{3} x_{3}-x_{3}\right) \\
& =\left(\mu_{3}-1\right)^{\alpha} B x_{3},
\end{aligned}
$$

thus $\left(\mu_{3}-1\right)^{\alpha} B x_{3} \geqslant \mu_{3}^{\alpha} B x_{3}$ and $\left[\left(\mu_{3}-1\right)^{\alpha}-\mu_{3}^{\alpha}\right] B x_{3} \doteq \alpha_{3} B x_{3} \in$ $P$. It also contradicts the condition (iii) in Definition 2 for $\alpha_{3}<0, B x_{3} \neq \theta$, and so the condition (L-S) is satisfied. Therefore, it follows from Lemma 3 that the conclusion of Theorem 11 holds.

Remark 12. (i) Theorem 11 is a generalization of the Petryshyn theorem.

(ii) In fact, Theorems 9 and 11 are actually the direct consequences because (8) and (10) immediately imply (5) due to $B: E \rightarrow P$.

Theorem 13. Suppose that $A: \bar{\Omega} \rightarrow E$ is a completely continuous operator. Let $P$ be a cone of $F$ and let the operator $B: E \rightarrow P$ be $\alpha$-positive-homogeneous operator such that $B x>\theta$ for $x \neq \theta$. If $\alpha>1$ and $B$ satisfies the following condition:

$$
B(A x+x) \leqslant B A x+B x, \quad \forall x \in \partial \Omega,
$$

then $A$ has at least one fixed point in $\bar{\Omega}$.

Proof. Suppose that (L-S) is not satisfied under the condition of (12). Then there exist $x_{4} \in \partial \Omega$ and $\mu_{4} \geqslant 1$ such that $A x_{4}=\mu_{4} x_{4}$. It is easy to see that $\mu_{4}>1$. Now, we consider the function defined by

$$
f(t)=t^{\alpha}+1-(t+1)^{\alpha}, \quad \forall t \geqslant 1, \alpha>1 .
$$

For $f^{\prime}(t)=\alpha t^{\alpha-1}-\alpha(t+1)^{\alpha-1}<0$, for all $t>1, \alpha>1$. So $f(t)$ is a strictly decreasing function in $[1,+\infty)$, and then $f(t)<f(1)=0$, for all $t>1$, or $t^{\alpha}+1<(t+1)^{\alpha}$, for all $t>1, \alpha>1$. Consequently, we can get from (12)

$$
\begin{aligned}
B\left(A x_{4}+x_{4}\right) & =B\left(\mu_{4} x_{4}+x_{4}\right) \\
& =\left(\mu_{4}+1\right)^{\alpha} B x_{4} \\
& \leqslant B A x_{4}+B x_{4} \\
& =B\left(\mu_{4} x_{4}\right)+B x_{4} \\
& =\mu_{4}^{\alpha} B x_{4}+B x_{4} \\
& =\left(\mu_{4}^{\alpha}+1\right) B x_{4},
\end{aligned}
$$

then $\left[\left(\mu_{4}^{\alpha}+1\right)-\left(\mu_{4}+1\right)^{\alpha}\right] B x_{4} \doteq \alpha_{4} B x_{4} \in P$, which contradicts the condition (iii) in Definition 2. Hence, the condition (L-S) is satisfied and the conclusion of Theorem 13 holds.

In the same way, if $0<\alpha<1$, then some new fixed-point theorems can be obtained by taking the inverse direction in the inequalities in conditions (5), (8), (10), and (12).

Theorem 14. Suppose that $A: \bar{\Omega} \rightarrow E$ is a completely continuous operator. Let $P$ be a cone of $F$, and let the operator $B: E \rightarrow P$ be $\alpha$-positive-homogeneous operator such that $B x>\theta$ for $x \neq \theta$. If $0<\alpha<1$ and $B$ satisfies one of the following conditions:

(i) $B(A x-x) \leqslant B A x-B x$, for all $x \in \partial \Omega$,

(ii) $B A x \geqslant B x$, for all $x \in \partial \Omega$,

(iii) $B A x \geqslant B(A x-x)$, for all $x \in \partial \Omega$,

(iv) $B(A x+x) \geqslant B A x+B x$, for all $x \in \partial \Omega$,

then $A$ has at least one fixed point in $\bar{\Omega}$.

Similarly, we can apply the above definitions and Lemma 4 to derive the following fixed point theorems.

Theorem 15. Suppose that $A: \bar{\Omega} \rightarrow E$ is a semiclosed 1-setcontractive operator. Let $P$ be a cone of $F$, and let the operator $B: E \rightarrow P$ be $\alpha$-positive-homogeneous such that $B x>\theta$ for $x \neq \theta$. If $\alpha>1$ and $B$ satisfies one of the following conditions:

(i) $B(A x-x) \geqslant B A x-B x$, for all $x \in \partial \Omega$,

(ii) $B A x \leqslant B x$, for all $x \in \partial \Omega$,

(iii) $B A x \leqslant B(A x-x)$, for all $x \in \partial \Omega$,

(iv) $B(A x+x) \leqslant B A x+B x$, for all $x \in \partial \Omega$,

then $A$ has at least one fixed point in $\bar{\Omega}$.

Theorem 16. Suppose that $A: \bar{\Omega} \rightarrow E$ is a semiclosed 1-setcontractive operator. Let $P$ be a cone of $F$, and let the operator $B: E \rightarrow P$ be $\alpha$-positive-homogeneous operator such that $B x>\theta$ for $x \neq \theta$. If $0<\alpha<1$ and $B$ satisfies one of the following conditions:

(i) $B(A x-x) \leqslant B A x-B x$, for all $x \in \partial \Omega$, 
(ii) $B A x \geqslant B x$, for all $x \in \partial \Omega$,

(iii) $B A x \geqslant B(A x-x)$, for all $x \in \partial \Omega$,

(iv) $B(A x+x) \geqslant B A x+B x$, for all $x \in \partial \Omega$,

then $A$ has at least one fixed point in $\bar{\Omega}$.

Remark 17. Because completely continuous operators belong to the class of semiclosed 1-set-contractive operators, Theorems 15 and 16 are more general than Theorems 7-14.

\section{Some Corollaries}

Now we will list some existing results in the references.

Corollary 18 (see [4]). Suppose that $A: \bar{\Omega} \rightarrow E$ is a semiclosed 1-set-contractive operator. If there exists $\alpha>1$ such that

$$
\|A x-x\|^{\alpha} \geqslant\|A x\|^{\alpha}-\|x\|^{\alpha}, \quad \forall x \in \partial \Omega,
$$

then $\operatorname{deg}(I-A, \Omega, \theta)=1$, and so A has at least one fixed point in $\bar{\Omega}$.

In fact, if we take the operator $B=\|\cdot\|^{\alpha}$ in Theorem 15 (i), the Corollary 18 can be obtained immediately. So it is the special case of our main results and also the following corollaries are similar.

Corollary 19 (see [5]). Suppose that $A: \bar{\Omega} \rightarrow E$ is a semiclosed 1-set-contractive operator. If $0<\alpha<1$ such that

$$
\|A x-x\|^{\alpha} \leqslant\|A x\|^{\alpha}-\|x\|^{\alpha}, \quad \forall x \in \partial \Omega,
$$

then $\operatorname{deg}(I-A, \Omega, \theta)=1$, and so $A$ has at least one fixed point in $\bar{\Omega}$.

Corollary 20 (see [4]). Suppose that $A: \bar{\Omega} \rightarrow E$ is a semiclosed 1-set-contractive operator. If $\alpha>1$ such that

$$
\|A x+x\|^{\alpha} \leqslant\|A x\|^{\alpha}+\|x\|^{\alpha}, \quad \forall x \in \partial \Omega,
$$

then $\operatorname{deg}(I-A, \Omega, \theta)=1$, and so A has at least one fixed point in $\bar{\Omega}$.

Corollary 21 (see [4]). Suppose that $A: \bar{\Omega} \rightarrow E$ is a semiclosed 1-set-contractive operator. If there exists $0<\alpha<1$ such that

$$
\|A x+x\|^{\alpha} \geqslant\|A x\|^{\alpha}+\|x\|^{\alpha}, \quad \forall x \in \partial \Omega,
$$

then $\operatorname{deg}(I-A, \Omega, \theta)=1$, and so A has at least one fixed point in $\bar{\Omega}$.

\section{Acknowledgments}

The authors would like to thank the referees for the thorough reading and excellent comments which led to the improvement of the original paper. This work was supported by the Special Funds of the National Natural Science Foundation of China (Grant no. 51227003), the National Nature Science Foundation of China (Grant nos. 10971179, 11026203), the Nature Science Foundation of Jiangsu Province (Grant no. BK2011202), and the Priority Academic Program Development (PAPD) of Jiangsu Higher Education Institutions.

\section{References}

[1] M. Altman, "A fixed point theorem in Banach space," Polish Academy of Sciences, vol. 5, pp. 89-92, 1957.

[2] G. Zhang, T. Zhang, and T. Zhang, "Fixed point theorems of Rothe and Altman types about convex-power condensing operator and application," Applied Mathematics and Computation, vol. 214, no. 2, pp. 618-623, 2009.

[3] G. Li, "The fixed-point index and the fixed-point theorems of $k$-set-contraction mappings," Proceedings of the American Mathematical Society, vol. 104, no. 4, pp. 1163-1170, 1988.

[4] G.-Z. Li, S.-Y. Xu, and H.-G. Duan, "Fixed point theorems of 1-set-contractive operators in Banach spaces," Applied Mathematics Letters, vol. 19, no. 5, pp. 403-412, 2006.

[5] S. Xu, "New fixed point theorems for 1-set-contractive operators in Banach spaces," Nonlinear Analysis. Theory, Methods \& Applications, vol. 67, no. 3, pp. 938-944, 2007.

[6] J. X. Sun and X. Y. Zhang, "A fixed point theorem for convexpower condensing operators and its applications to abstract semilinear evolution equations," Acta Mathematica Sinica. Chinese Series, vol. 48, no. 3, pp. 439-446, 2005.

[7] R. D. Nussbaum, "Degree theory for local condensing maps," Journal of Mathematical Analysis and Applications, vol. 37, pp. 741-766, 1972.

[8] R. D. Nussbaum, "The fixed point index and asymptotic fixed point theorems for $k$-set-contractions," Bulletin of the American Mathematical Society, vol. 75, pp. 490-495, 1969.

[9] J. X. Sun, Nonlinear Functional Analysis and Its Application, Science Press, Beijing, China, 2007, Chinese.

[10] H. Amann, "Fixed point equations and nonlinear eigenvalue problems in ordered Banach spaces," SIAM Review, vol. 18, no. 4, pp. 620-709, 1976.

[11] D. J. Guo and J. X. Sun, "The calculation of topological degree and its applications," Journal of Mathematical Research and Exposition, vol. 8, no. 3, pp. 469-480, 1988.

[12] W. V. Petryshyn, "Remarks on condensing and $k$-set-contractive mappings," Journal of Mathematical Analysis and Applications, vol. 39, pp. 717-741, 1972. 


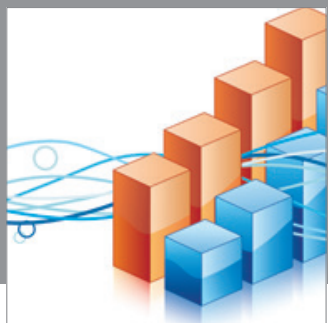

Advances in

Operations Research

mansans

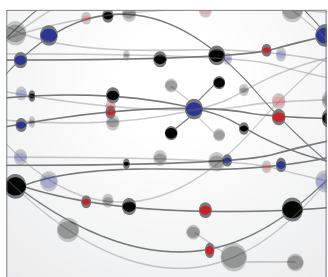

The Scientific World Journal
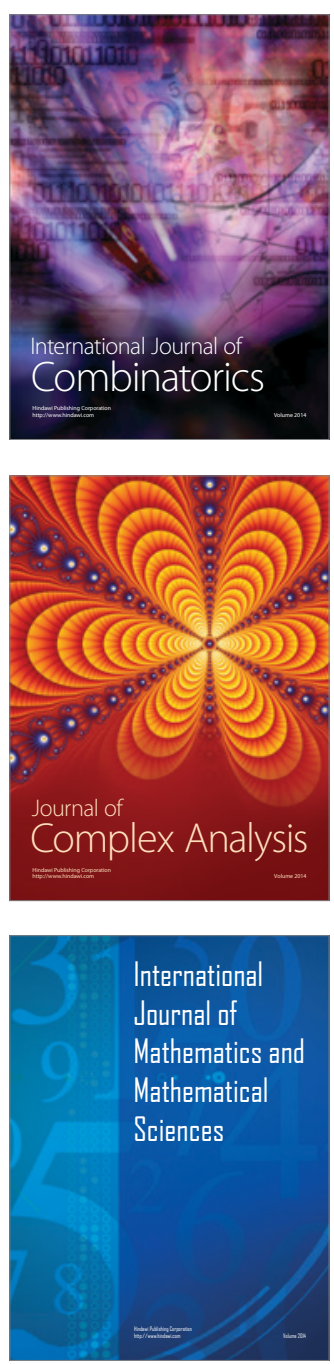
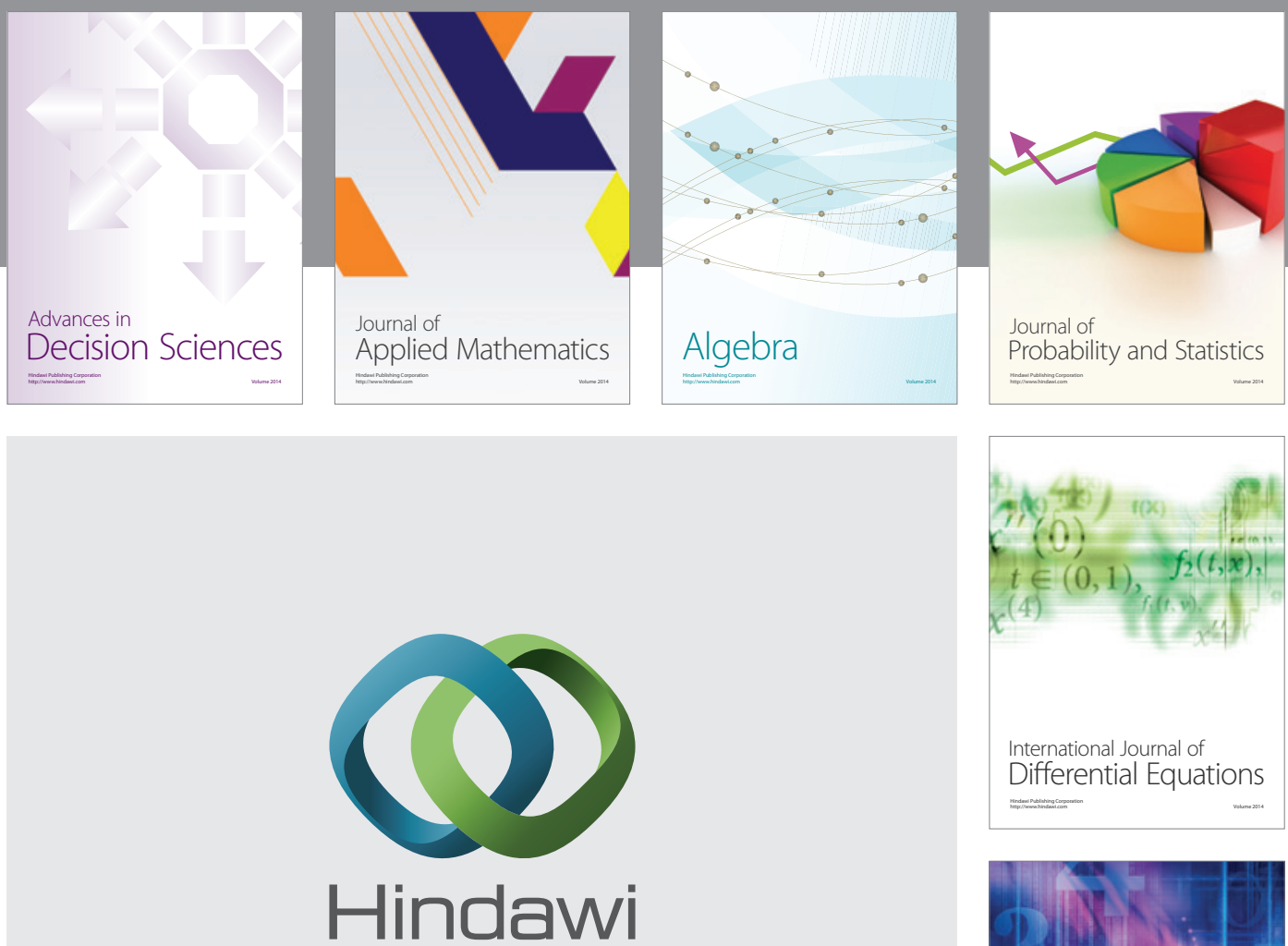

Submit your manuscripts at http://www.hindawi.com
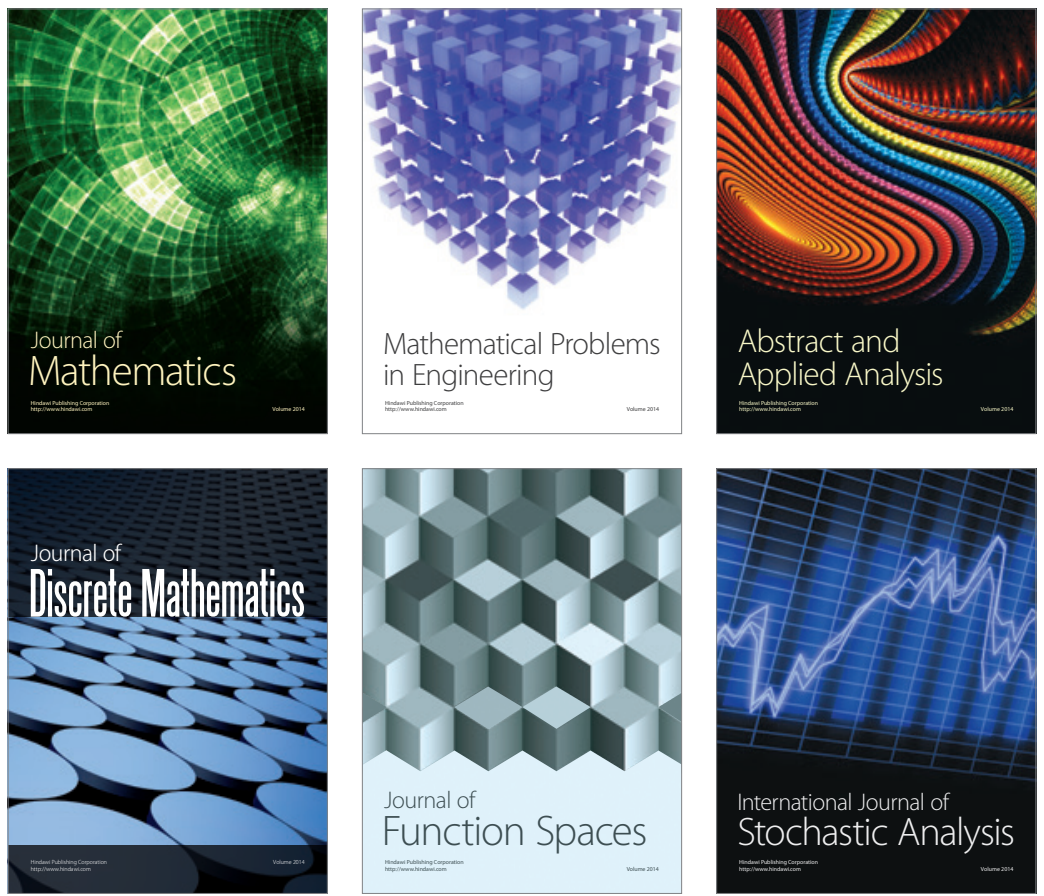

Journal of

Function Spaces

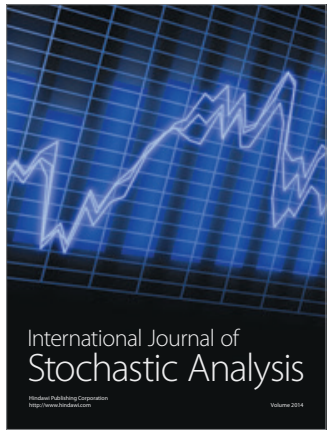

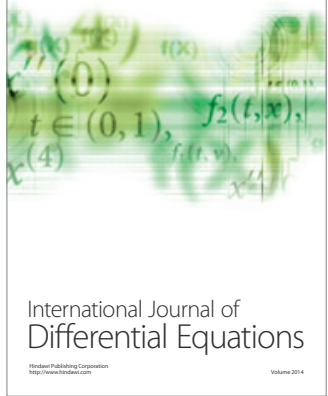
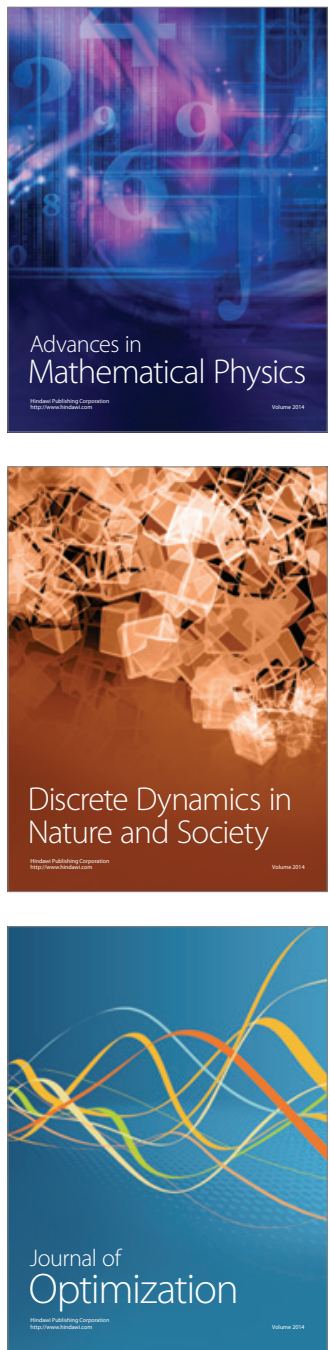